\title{
CAPACITY BUILDING FOR GEO-INFORMATION DRIVEN EMERGENCY RESPONSE IN CHINA
}

\author{
Zhongxiang Wang ${ }^{1}$, Hao $\mathrm{Wu}^{1}{ }^{1} *$, Jie Zhu ${ }^{1}$, Ronghui Zhang ${ }^{1}$, Hao Song ${ }^{2}$, Junwei Wang ${ }^{3}$, Zihao $\mathrm{He}^{4}$ \\ ${ }^{1}$ National Geomatics Center of China, Lianhuachi West Road 28, 100830 Beijing, China - \\ (wangzhongxiang, wuhao, zhujie, zhangronghui)@ngcc.cn \\ ${ }^{2}$ HeiLongjiang Geomatics Center of MNR, 150081, Harbin, China-8465509@qq.com \\ ${ }^{3}$ Sichuan Surveying and Mapping Geographic Information Bureau Surveying and Mapping Technology Service Center, 610081 \\ Chengdu, China - scienceandtech@126.com \\ ${ }^{4}$ The First Geodetic Surveying Brigade of MNR, 710054 Xi'an, China - strive_he@163.com
}

KEY WORDS: Emergency Response, Geo-information, UAV Aerial Photogrammetry, Automatic Processing, Service platform

\begin{abstract}
:
Surveying and mapping technologies played a significant role in former emergency response activities. Despite remarkable progress on emergency surveying and mapping has been achieved in China, there are still some limits to meet the quick and smart response requirements. Aiming to fill the gaps, Chinese government has proposed a capacity building project for geo-information driven emergency response. In this paper, the business process and data flow of emergency surveying and mapping are analysed firstly. Then, the major design of this project is introduced through 4 parts. These are capacity of UAV aerial photography, capacity of field surveying, capacity of smart service and capacity of data integration. Finally, the preliminary progress and prospective achievement are discussed. The implementation of this project could be an example for comprehensive application of advanced surveying and mapping technologies, and could also be a basis to improve our national emergency response framework.
\end{abstract}

\section{INTRODUCTION}

Natural disasters occurred frequently in China and have caused serious losses to people's lives and property (LIU et al., 2018). Taking Sichuan province in where Wenchuan earthquake has occurred in 2008 as an example, there are more than 40,000 potential geological disasters points been confirmed and about $1,650,000$ people are still being affected by them. When disasters occurred, we need timely and accurate information to support the decision making and rescue operations. Surveying and mapping technologies, which could be used to obtain the image of disaster areas and further analyse the damage (ZHU et al., 2014; NING et al., 2016), have made powerful contributions to former emergency response activities and became one of the important parts of national emergency response framework in China (MIN 2013; ZHOU et al., 2015).

The concept of emergency surveying and mapping was adopted widely after Wenchuan earthquake in China. It refers to the activities comprehensively using remote sensing, geoinformation, GNSS (Global Navigation Satellite System) and communications technology to provide surveying and mapping service for emergency prevention and response.

While, in the area of research, it has been a hot topic from about 30 years ago. In the early stage, limited by the development of surveying and mapping technologies, researchers mainly focused on using geo-information for emergency mapping and analysis. With the rapid expansion of remote sensing and processing, it was possible for us to obtain images before and after disaster more easily. Image processing and analysis for emergency response have become a hot area for about 20 years (Mantovani et al., 1996; Arnous et al, 2011; Williams et al,
2018). For example, Mantovani et al. introduced remote sensing technologies for landslide studies and hazard zonation in Europe in 1996. Williams et al. used optical imagery for rapid landslide assessment in 2015 Nepal earthquakes. They also pointed out that a big challenge for emergency response based on satellite image is how to collect useable image in time and it needs close cooperation of different governments and originations. Actually, restricted by the fix visit circle and cloud coverage of satellite remote sensing, it is difficult to get available image immediately. To solve this problem, many researchers attempted to use VGI (Volunteer Geographic Information), UAV (Unmanned Aerial Vehicle) and web service technologies to collect the information of disaster area. For example, in the Haitian Earthquake, CrisisCamp et al. provided a web service platform for volunteer to label the information for streets been destroyed and people waiting for rescue (Zook et al, 2010). Zhai et.al proposed a sensor web and web service-based approach for active hydrological disaster monitoring, and provided an application to demonstrate the benefits of the proposed approach over the traditional approach. Lei et.al analysed the application based on UAV remote sensing for monitoring secondary geological disasters after earthquakes. on the other hands, some countries have engaged in build national emergency surveying and mapping response system. For example, USA has established emergency management department in federal government, state and cities. NASA has brought forward NASA's Earth Science Disaster Response Team, which included multi-Center and institutional efforts to map damage and flooding - for Florence, in-person support at FEMA HQ to better understand their immediate and longerterm remote sensing needs (Andrew and Jordan, 2019). Japan

\footnotetext{
* Corresponding author
} 
has established an emergency management team composed of both government and social organization to response to national and regional disaster (Cao et al, 2018).

Surveying and mapping and other related technologies have made great progress in last decade. There are more and more satellites could be selected to obtain image of disaster area. Industrial grades UAVs, with longer endurance and heavier mission payload, have been applied into aerial photogrammetry area. With the improvement of variety and accurate of aerial sensors, the size and weight of them are becoming more lightweight. The development of deep learning, big data and knowledge service technologies have made it possible to realize automatic and collaborative processing of disaster geoinformation.

While, due to the scattered distribution of disasters in China, the current equipment and methods are lack of capacity in fill the requirements of quick response. The gaps demonstrated as 4 aspects: 1) the speed of image obtaining is not quick enough for decision making; 2) the equipment for gathering multiple geoinformation in frontline is not effective enough to fill the needs of disaster monitoring; 3 ) the software used for conventional 4D product are not appropriate for the automatically processing of disaster geo-information; 4) the transmission and sharing of disaster geo-information are still restricted by missing of interconnected network and uniform platforms. Therefore, make use of the new technologies to improve the current capacity for emergency surveying and mapping is imperative.

Taking the capacity building project of national emergency response in China as background, the aim of this research is establishing a collaborative and dynamic emergency surveying and mapping system.

\section{BUSINESS PROCESS ANALYSIS OF EMERGENCY SURVEYING AND MAPPING}

For emergency incidents, the emergency surveying and mapping business process can be divided into four links: on-site rapid aerial emergency surveying and mapping, rapid processing of emergency surveying and mapping, rapid sharing of emergency surveying and mapping products, and emergency frontline rapid support service. The overall business process of emergency surveying and mapping is shown in Figure 1.

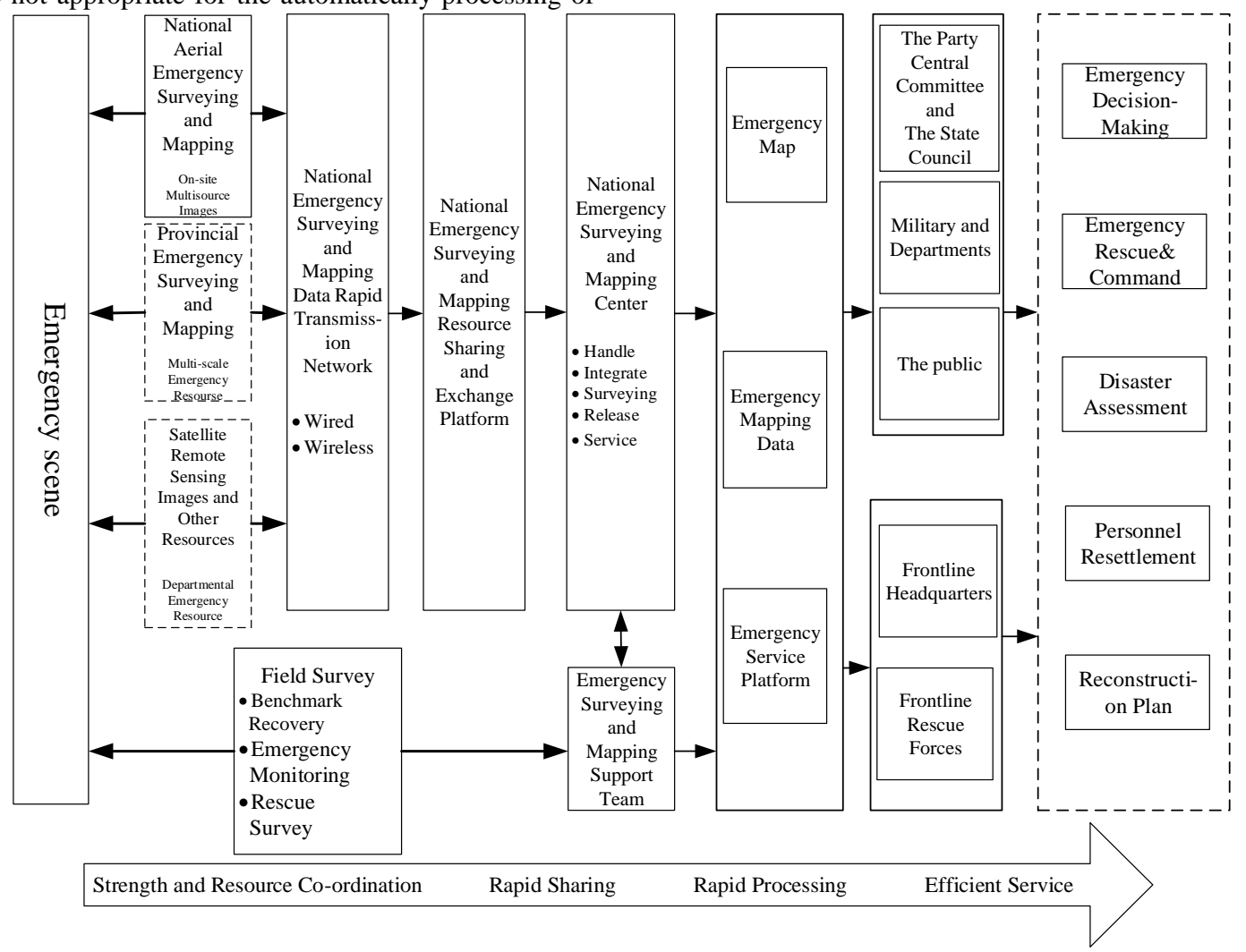

Figure 1. National emergency surveying and mapping business flow chart

\subsection{On-site rapid aerial emergency surveying andmapping}

It is the primary core task of national emergency surveying and mapping to obtain real disaster information at the first time. The main task is to make full use of the established emergency aerial mapping capabilities in China, and adopt appropriate airborne remote sensing technology for different types of emergencies, to quickly implement on-site information acquisition, and to ensure that the scene images in the area affected by emergencies are acquired at the first time. And it provides emergency mapping support for emergency response, scientific assessment of disasters, and effective implementation of emergency rescue.
Considering the factors such as the weather, terrain, and size of the emergency, on-site rapid aerial emergency surveying and mapping requires an all-day, high-efficiency acquisition capability.

\subsection{Emergency frontline rapid support servicea}

After a major emergency, according to the national emergency response, it is necessary to immediately dispatch the National Emergency Surveying and Mapping Support Team to the emergency site to provide emergency surveying and mapping support, which includes frontline field information collection, secondary and derivative disaster monitoring, rapid processing 
and producing of emergency mapping products. The products include large-scale topographic map data, secondary disaster monitoring data and other emergency disaster map data, as well as emergency thematic drawing and other emergency mapping products. Thus, it will ensure the smooth development of frontline emergency command, lifeline repair and rescue, engineering rescue, personnel search and rescue, personnel resettlement and construction.

\subsection{Rapid processing of emergency surveying and mapping}

A large amount of original image obtained through on-site emergency aerial mapping must be processed by mapping technology before being used by emergency departments. Due to the requirements of emergency disposal aging, rapid processing of emergency surveying and mapping requires minimal processing time. Therefore, it is necessary to establish an integrated and efficient processing capability for rapid processing of emergency surveying and mapping, and to quickly produce emergency mapping products required for different emergency phases, including emergency maps, emergency surveying and mapping data, emergency service platforms. Based on it, the disaster situation will be analysed and judged step by step, and the rescue force will be scientifically dispatched. As a result, it is able to provide emergency mapping service to the relevant leadership of the Party Central Committee and the State Council, as well as to the national emergency management department and various specialized emergency departments.

\subsection{Rapid sharing of emergency surveying and mapping products}

Through the emergency mapping resources sharing nodes and the comprehensive use of wired and wireless network transmission, a nationwide emergency surveying and mapping resource sharing network is constructed, which covers the whole country and all aspects of emergency mapping. The sharing network makes use of provincial emergency surveying and mapping resource and on-site multi-source information obtained from various departments, establishing a national emergency surveying and mapping resource database, and provides an integrated and standardized data foundation for national emergency surveying and mapping support. In addition, a shared exchange platform is established which could provide fast, efficient and smooth emergency service for the Party Central Committee, the State Council and the emergency management department.

\section{DATA FLOW ANALYSIS OF EMERGENCY SURVEYING AND MAPPING}

According to the use of emergency map, the type of emergency services can be divided into: service based on existing emergency surveying and mapping resources, service based on satellite imagery, service based on aerial remote sensing images, service based on emergency frontline field surveying, etc. According to the type and level of emergencies, the national emergency mapping response chooses one or more of the above services to timely carry out emergency mapping support services. The data flow of the above four types of emergency mapping service types is shown in Figure 2.

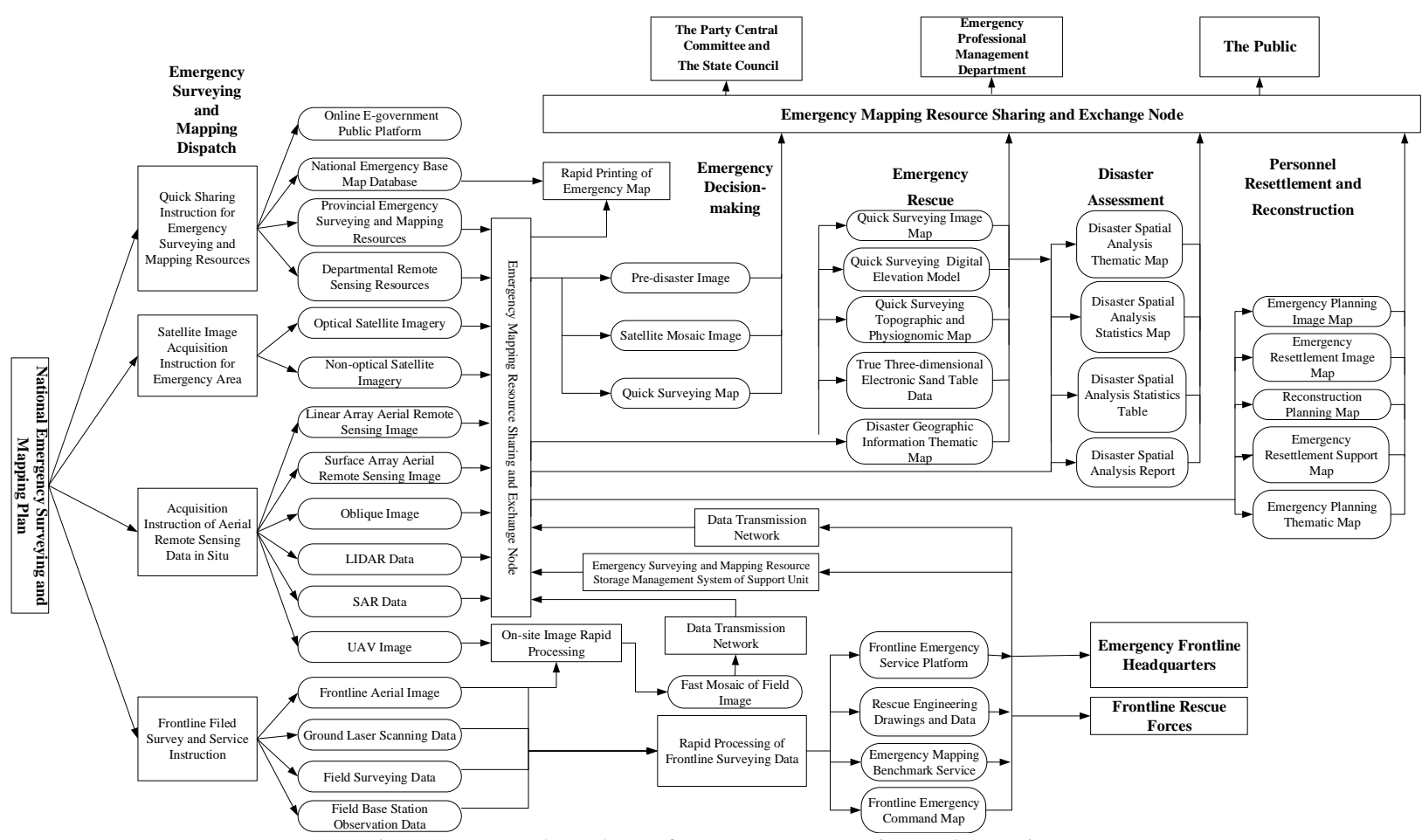

Figure 2. Data Flow chart of emergency surveying and mapping

\subsection{Support service data flow based on emergency} surveying and mapping resource sharing

Using the national emergency base map database, provincial emergency surveying and mapping data, departmental remote 
sensing resources, online e-government and public platform and so on, three aspects of emergency service support work will be carried out immediately after receiving emergency mapping instructions. First, a green channel is provided for acquisition of emergency surveying and mapping product. The second is to carry out production of emergency geographic information. The third is to load emergency surveying and mapping products and update service in emergency e-government and public platform. Various collected and produced thematic maps, catalogue data are urgently loaded into the emergency e-government and public platform. The emergency surveying and mapping data are also informed in detail to relevant departments.

\subsection{On-site satellite image acquisition and support service data flow}

Satellite remote sensing imagery is one of the important data of emergency surveying and mapping products. After receiving emergency instruction, the domestic satellite image acquisition programming is carried out in time, the satellite attitude is adjusted, the satellite image of the emergency area is quickly collected. At the same time, foreign commercial satellites are contacted in time to maximize the acquisition of various on-site satellite image resources. Four aspects of work are carried out. Firstly, fast processing is used to produce quick-report image. Second, the rapid production of emergency mapping data is produced. Third, the production of disaster extraction and analysis are produced. Fourth, the production of emergency reconstruction planning data is produced.

\subsection{On-site aerial image acquisition and support service data flow}

Aerial photography is the most important technology and means to obtain comprehensive and detailed information on the emergency scene. For major emergencies, high-resolution aerial imagery materials are needed to quickly obtain real and detailed damage on site. It mainly includes the following data processing links. One is to carry out aerial photography in emergency area. The second is the rapid processing of UAV images with the National Aerial Emergency Surveying and Mapping Base. The third is to produce emergency map for emergency command and rescue purposes. The fourth is to carry out emergency geographic information extraction and analysis. The fifth is to carry out comprehensive emergency support services. The sixth is to carry out database management and safe backup of emergency surveying and mapping products.

\subsection{Emergency frontline field surveying support service data flow}

For emergencies requiring on-site support, the National Emergency Mapping Support Team will quickly assemble the relevant team members after receiving instructions. After equipment checking and testing, and existing emergency maps preparing, the team members go to the frontline to provide onsite field surveying support services for the emergency frontline headquarters and frontline rescue forces. There are mainly three aspects of the work. The first is to quickly organize on-site surveying and support service work. The second is to urgently restore the geodetic benchmark service in emergency areas. The third is to ensure the sharing of emergency mapping resources.

\section{OVERALL ARCHITECTURE DESIGN OF EMERGENCY SURVEYING AND MAPPING}

According to the analysis of emergency mapping business process, the main contents of the national emergency surveying and mapping capacity building project can be divided into four parts: capacity of UAV aerial photography, capacity of field surveying, capacity of smart service and capacity of data integration. The overall structure is shown in Figure 3:

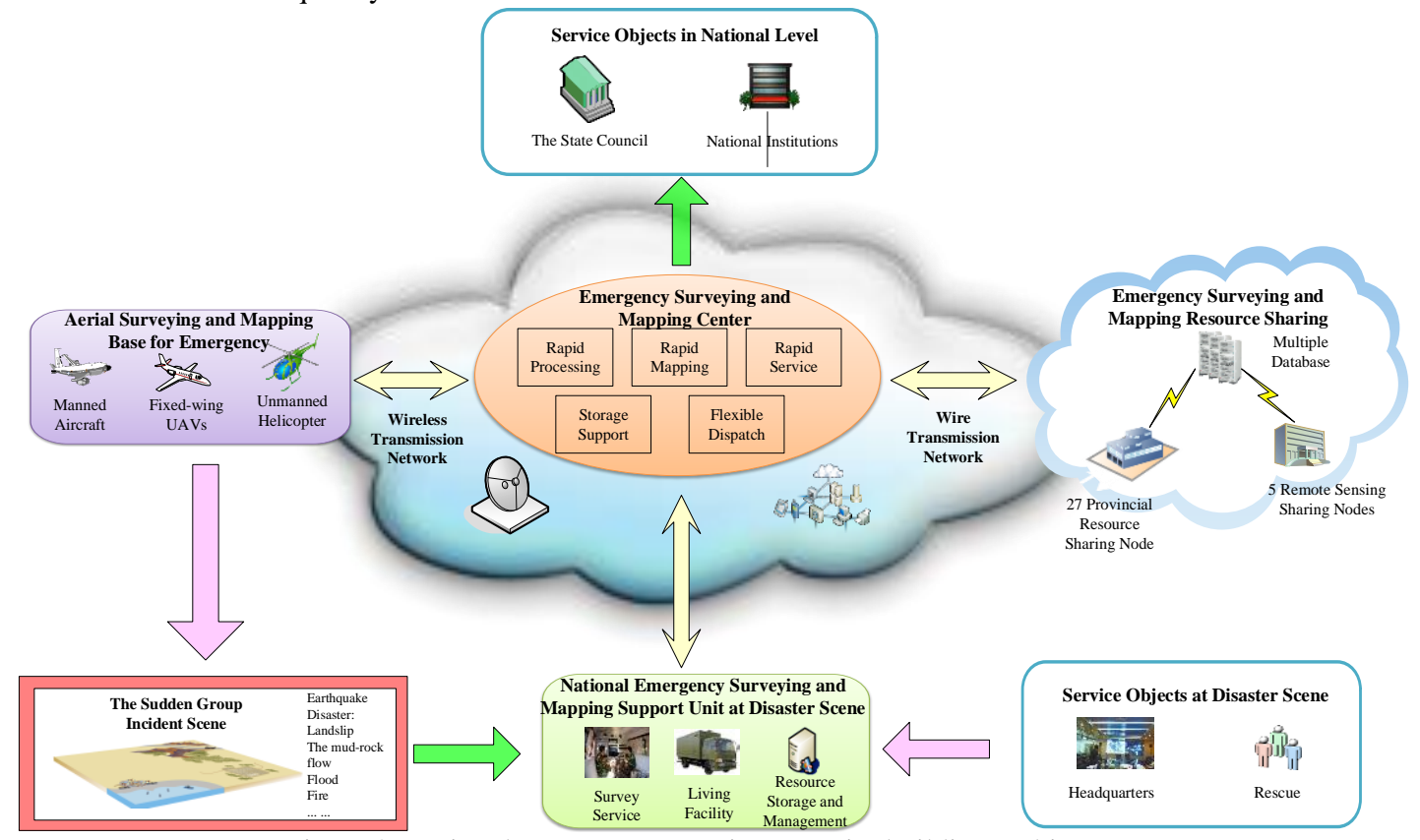

Figure 3. National emergency mapping capacity building architecture

\subsection{Capacity of UAV aerial photography}

The capacity building contents include middle-endurance fixedwing UAV aerial emergency surveying and mapping system, 
short-endurance fixed-wing UAV aerial surveying and emergency mapping system and unmanned helicopter aerial emergency surveying and mapping system, focusing on solving the rapid, comprehensive and dynamic acquisition of emergency on-site image, and improving the rapid acquisition capability and maneuverability. The manned aircraft emergency aerial surveying and mapping system which makes full use of the existing military resources is no longer built.

\subsection{Capacity of field surveying}

The capacity building contents include multi-function emergency work cabin, emergency field survey vehicle, and emergency data storage management system, focusing on solving standardization, integration and high efficiency of national emergency surveying and mapping frontline equipment, and improving the comprehensive and fast capability of emergency surveying and mapping teams. Conventional measuring instruments which use existing resources are no longer constructed.

\subsection{Capacity of smart service}

The capacity building contents include rapid processing of emergency surveying and mapping, rapid printing of emergency maps, rapid service of emergency surveying and mapping, emergency surveying and mapping storage support, flexible dispatch of emergency surveying of mapping. It focuses on providing emergency surveying and mapping support services for the Party Central Committee, the State Council, emergency management and rescue departments, and improving the ability of rapid processing, efficient management and diversified services of emergency mapping data.

\subsection{Capacity of data integration}

The capacity building contents include national emergency base map database system, emergency surveying and mapping resource sharing platform and emergency surveying and mapping data rapid transmission network. It focuses on solving the problem of poor, unpleasant and repetitive data sharing of national emergency surveying and mapping resources, and improving the real-time online sharing capabilities and ondemand sharing capabilities of emergency mapping resource sharing node.

\section{INTERFACE DESIGN OF EMERGENCY SURVEYING AND MAPPING SYSTEM}

According to the above framework, the flow chart among systems of the national emergency mapping support capacitybuilding project is shown in Figure 4.

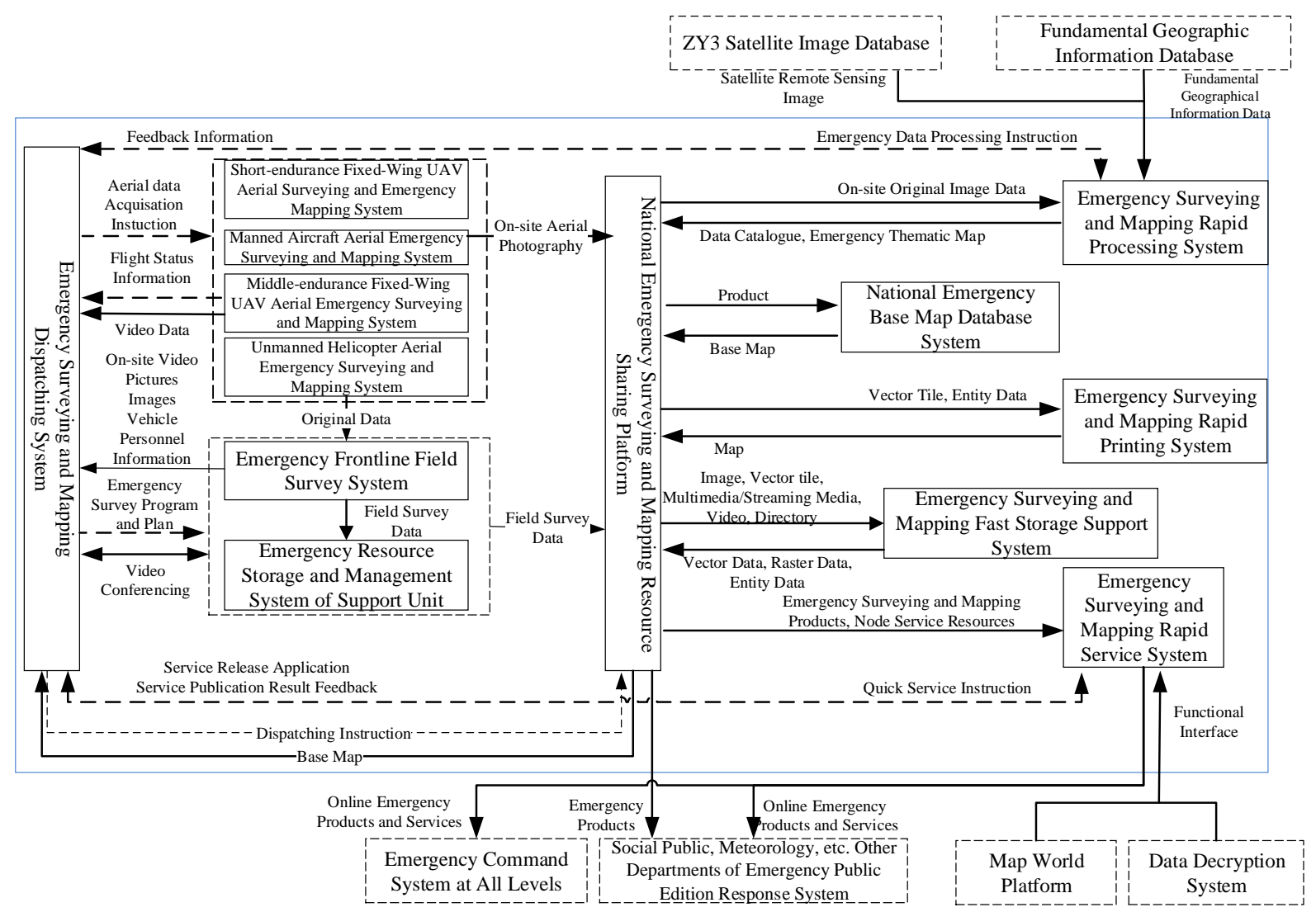

Figure 4. Flow design of emergency surveying and mapping system

(1) Emergency surveying and mapping dispatching system, as the backbone of emergency surveying and mapping, sends instructions to other systems and receives feedback after the completion of emergency surveying and mapping tasks. At the same time, the emergency surveying and mapping and dispatching system receives the base map data from the sharing platform, the real-time video data from the aerial emergency surveying and mapping system, and the urgently needed images from the emergency frontline field surveying system, and conducts video consultation with frontline.

(2) Emergency surveying and mapping data rapid transmission network, which is the link between emergency surveying and 
mapping capabilities, provides a supporting environment for other systems.

(3) Emergency surveying and mapping storage support system provides a backup and storage environment for the emergency surveying and mapping rapid processing system, the rapid printing system, the rapid service system, and the national emergency surveying and mapping resource sharing platform.

(4) Manned aircraft aerial emergency surveying and mapping system, in which middle-endurance fixed-wing UAV aerial emergency surveying and mapping system, short-endurance fixed-wing UAV system and unmanned helicopter aerial emergency surveying and mapping system are the main means of on-site information acquisition. Images and products are transmitted to the resource sharing platform and the dispatching system, by the transmission network and under the instruction of the emergency surveying and mapping dispatching system.

(5) Emergency frontline field survey system, which receives the instructions and emergency plan from emergency surveying and mapping dispatching system, obtains on-site ground information, and receives the aerial remote sensing image and product transmitted by the unmanned helicopter.Based on the pre-installed data from emergency resource storage and management system of support team, the on-site ground information is processed and stored. At the same time, the onsite ground information is transmitted to national emergency surveying and mapping resources sharing platform, by using national emergency surveying and mapping data rapid transmission network. Live video image, vehicle personnel information and needed images are transmitted to emergency surveying and mapping dispatching system, by the national emergency communication network. In addition, online video consultation with emergency surveying and mapping dispatching system is conducted through the national emergency communication network.

(6) Emergency resource storage and management system of support team is used to store the fundamental survey data and the base map in support areas. It provides base map support for the emergency frontline field survey system and provides storage support for the frontline on-site surveying. It can also be used as the resource node of provincial emergency surveying and mapping, and access to the national emergency surveying and mapping resource sharing platform.

(7) Emergency surveying and mapping rapid processing system, obtains pre-disaster satellite image and fundamental geographic information data from the existing ZY3 satellite image database and fundamental geographic information database, according to the instruction of the emergency surveying and mapping and dispatching system. On-site emergency image is obtained from the national emergency surveying and mapping resource sharing platform, the man-made aerial emergency surveying and mapping system, the middle-endurance and short-endurance fixed-wing UAV aerial emergency mapping system, the unmanned helicopter aerial emergency mapping system. Which is along with the data from emergency frontline field survey system, are transmitted to the processing system. Then after the production of emergency mapping results is completed, it is provided to the national emergency surveying and mapping resource sharing platform to serve as emergency products for emergency surveying and mapping rapid service and rapid printing system.

(8) Emergency surveying and mapping rapid service system, which uses the national emergency surveying and mapping data transmission network, obtains emergency surveying and mapping products and services through the national emergency surveying and mapping resource sharing platform, and provides online emergency mapping and geographic information services, according to the instruction of the emergency surveying and mapping and dispatching system.

(9) Emergency surveying and mapping rapid printing system, which receives the emergency surveying and mapping base data and on-site image provided by the national emergency surveying and mapping resource sharing platform, generates emergency thematic maps, provides cloud printing services and electronic map services, according to the instruction of the emergency surveying and mapping and dispatching system.

(10) National emergency surveying and mapping resource sharing platform is responsible for data sharing and exchange within and outside the system. It integrates and manages emergency base data, on-site emergency map, and production results of system. The objects of management mainly include aerial emergency original image obtained from include manned aircraft aerial emergency surveying and mapping system, middle-endurance and short-endurance fixed-wing UAV aerial emergency surveying and mapping system, the unmanned helicopter aerial emergency surveying and mapping system, and include results of surveying and mapping in different emergency stages, geographic information extraction and analysis products, acquisition and processing result of emergency frontline field survey system. According to the instruction of the emergency surveying and mapping and dispatching system, the above emergency surveying and mapping results should be distributed to the emergency department of the State Council, the disaster assessment management department and the emergency departments of other ministries and commissions.

(11) National emergency base map database system, which stores emergency electronic maps, emergency thematic data, etc., provides base map for emergency surveying and mapping rapid printing system and emergency surveying and mapping rapid service system through the sharing platform according to the instruction of the emergency surveying and mapping and dispatching system.

\section{CONLUSIONS}

Based on the above design, the main contents of this capacity building project will be completed in this year. It is believed that great advance could be expected through the implementation of this project. A preliminary estimation indicates that when this project was completed, we could get $80 \%$ of land area in China within 4 hours after the UAVs have been launched, and the multiple products of disaster area could be provided within 12 hours. But the establishment of emergency surveying and mapping system is a huge project. It involved not only technologies and equipment. We still need to work on the operational model and collaborative mechanism.

\section{REFERENCES}

Andrew Molthan, Jordan Bell. NASA Earth Science Activities Supporting Analysis and Response to Hurricanes Florence and Michael [EB/OL]. 2019-01-07[2019.06.01] https://ntrs.nasa.gov/archive/nasa/casi.ntrs.nasa.gov/201900000 77.pdf.

Arnous M O. Integrated remote sensing and GIS techniques for landslide hazard zonation: a case study Wadi Watier area, South Sinai, Egypt. J COAST CONSERV, 2011, 15(4):477-497.

Cao J, Zhu L, Han H, et al. Emergency Disaster Management. Modern Emergency Management. Springer, Singapore, 2018: 255-290.

Lei T, Zhang Y, Wang X, et al. The application of unmanned aerial vehicle remote sensing for monitoring secondary 
geological disasters after earthquakes[C]// Ninth International Conference on Digital Image Processing (ICDIP 2017). International Society for Optics and Photonics, 2017.

LIU Jiping, ZHANG Yongchuan, XU Shenghua, ZHANG Fuhao, WANG Yong, ZHU Yi, LIU Chunyang. Top-Level Design Study for the Integrated Disaster Reduction Intelligent Service. GEOMATICS AND INFORMATION SCIENCE OF WUHAN UNIVERS, 2018, 43(12): 2250-2258.(In Chinese)

Mantovani F, Soeters R, Westen C J V . Remote sensing techniques for landslide studies and hazard zonation in Europe [J]. Geomorphology, 1996, 15(3):213-225.

MIN Yiren. Give Full Play to Advantages and Continue to Promote Emergency Surveying and Mapping Guarantee Work. China Surveying and Mapping, 2013(4):6-9. (In Chinese)

NING Jinsheng, WANG Zhengtao. The Transformation, Upgrading and Development of Surveying \& Mapping and Geographic Information Science and Technology. Geospatial Information, 2016, 14(2):1-5. (In Chinese)

SHI Xiaoming, ZHANG Tianfeng, WANG Yang, CHEN Qing. Application of Medium Endurance Fixed-wing UAV on the Construction of Aviation Emergency System of Surveying and Mapping in Hubei Province. Bulletin of Surveying and Mapping, 2017(S1):191-193+197. (In Chinese)

Williams J G , Rosser N J , Kincey M E , et al. Satellite-based emergency mapping using optical imagery: experience and reflections from the 2015 Nepal earthquakes. NAT HAZARD EARTH SYS, 2018, 18(1):185-205.

YANG Yan, DU Ganlin, CAO Qitong. Application of UAV Aerial Surveying Technology in Geological Disaster Emergency Mapping. Bulletin of Surveying and Mapping, 2017(S1):125128. (In Chinese)

Yusoff A R, Darwin N, Majid Z, et al. Geospatial-Based Slope Mapping Studies Using Unmanned Aerial Vehicle Technology[C]//GeoInformation for Disaster Management Conference. Springer, Cham, 2018: 185-208.

Zhai, X.; Yue, P.; Zhang, M. A Sensor Web and Web ServiceBased Approach for Active Hydrological Disaster Monitoring. ISPRS Int. J. Geo-Inf. 2016, 5, 171.

ZHOU Zhiwu, ZHAO Yong, ZHU Xiuli, ZHAO Tingting, LI Ran, ZHU Jie. Surveying and Mapping Emergency Support Services Provided by NGCC:Present and Future. Bulletin of Surveying and Mapping, 2015(10):16-19. (In Chinese)

ZHU Qing, CAO Zhenyu, LIN Hui, XIE Weiting, DING Yulin. Key Technologies of Emergency Surveying and Mapping Service System. GEOMATICS AND INFORMATION SCIENCE OF WUHAN UNIVERS, 2014, 39(5): 551-555. (In Chinese)

ZHU Qing, HAN Huipeng, YU Jie, DU Zhiqiang, ZHANG Junxiao, WU Chen, SHEN Fuqiang. Multi-objective Optimization Scheduling Method for UAV Resources in Emergency Surveying and Mapping. GEOMATICS AND INFORMATION SCIENCE OF WUHAN UNIVERS, 2017, 42(11): 1608-1615. (In Chinese)

Zook M , Graham M , Shelton T, et al. Volunteered Geographic Information and Crowdsourcing Disaster Relief: A Case Study of the Haitian Earthquake. World Med Health Policy, 2010, 2(2):7-33. 